\title{
ADEQUATE SAMPLING, AN IMPORTANT STEP TOWARDS RELIABLE DECISIONS BASED ON ANALYTICAL RESULTS
}

\author{
Lennart Mathiasson \\ Lund University, Sweden \\ Lennart Mårtensson \\ Kristianstad University, Sweden
}

\begin{abstract}
The efforts spent on judgments of how to perform sampling are often small compared to other steps in the analytical procedure. As a result decisions may be based on irrelevant results. If the sampling is intended to provide information how to reduce and control environmental pollution, it is important to design the sampling strategy according to scientific principles. Sampling will be discussed in relation to determinations of organic as well as inorganic species in different types of polluted matrices, .e.g. water, sediments and air. Examples will be taken from our own research projects about treatment processes for contaminated matrices.. Accurate sampling involves a basic planning including objectives, cost effectiveness versus the budget, variability of contamination leading to statistical considerations, site accessibility and robustness of the used devices. A strategy for the sampling, often in a form of a scheme, is needed. The Laqua Protocol developed within our research projects will be presented. This protocol is flexible and dynamic and can be altered and optimized based on the demands at the individual sampling site. Sampling of complicated matrices often creates problems with interfering agents and breakdown of unstable analytes. In this type of samples the concentrations of analytes are generally several orders of magnitudes lower than the concentrations of major constituents in the sample. Accordingly the advantages of an early work-up, preferably in connection with a sampling procedure, is paid especially attention
\end{abstract}

\section{KEYWORDS}

Sampling, strategy, protocol, sample work-up, statistics, contaminants

\section{INTRODUCTION}

It is vital to firstly consider the objective of a project before any judgement of sampling procedure and choice of sampling equipment is made. For example, if results are to be used for any type of mass-balance calculation or to establish the possibility for high peak concentrations the choice of sampling procedure must be different. In favourable cases a long series of practical achievements and experiences of similar problems has made a protocol available, which can facilitate the design of the actual sampling procedure. For example when 
studying leachate waters from landfills a protocol has been established, which include different parameters to be considered when dealing with such environmental problems. In figure 1 this protocol is visualised.

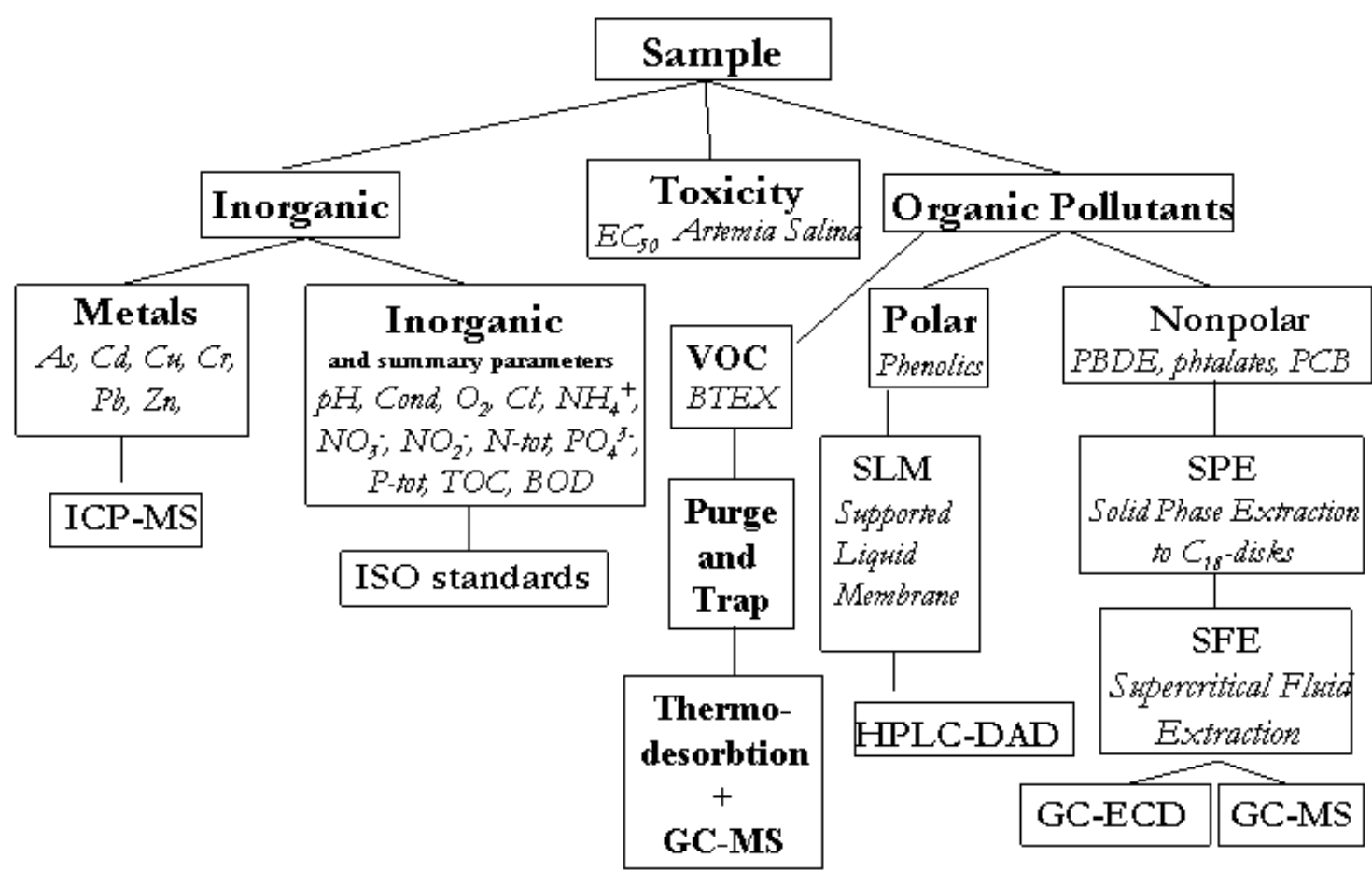

Figure 1. The Laqua protocol [1].

From figure 1 it is clear that direct instrumentation is desirable for measuring such parameters that can be changed during sampling storage as $\mathrm{pH}$ and conductivity. It is a important to realise that different types of materials in sample containers can be needed for metals and organic pollutants, respectively. Other things to consider is that different preservation procedure may be necessary, e.g. for metals and organic pollutants. This paper discusses different modes and techniques for sampling and features that influence the choice of procedure.

\section{SAMPLING}

\subsection{Sampling mode}

There are different sampling procedures that fulfil different needs, like grab sampling, intermittent sampling and time-integrated sampling (figure 2). The two latter often take in to consideration variation in flow-rate with time (flow proportional sampling). However, even in grab sampling it is important to know the flow rate. In grab sampling it is possible, using a 
frequent sampling, to get a reliable variation in concentration of the analyte with time. The draw-back is of course that the cost will increase as a consequence of the large number of samples taken. Time integrated sampling offer a good possibility to determine average concentrations useful for mass-balance calculations to a substantially lower cost. Since frequent grab sampling leads to large costs the number of samples taken are usually too small for reliable estimation of average concentration see table 1 below

Table1 . Determination of 2-methyl-4-chlorophenoxyacetic acid (MCPA) in a Sweden brook for a period of 10 days. * [2].

Concentration of MPCA (ppb)

\begin{tabular}{lcccccccccc}
\hline Day: & 1 & 2 & 3 & 4 & 5 & 6 & 7 & 8 & 9 & 10 \\
\hline $\begin{array}{l}\text { Integrating } \\
\text { sampling }\end{array}$ & 2.1 & 0.1 & 0.3 & 0.1 & 0.2 & 0.1 & 0.6 & 4.5 & 0.7 & 0.2 \\
$\begin{array}{l}\text { Grab } \\
\text { sampling }\end{array}$ & 0.2 & 0.3 & 0.1 & 0.1 & 0.1 & 0.1 & 1.5 & 1.2 & 1.2 & 0.1
\end{tabular}

*Values obtained either from integrating sampling using the membrane technique for a period of one day. Or from grab samples taken each morning.

From table 1 it seems clear that in grab sampling there is risk of underestimation of the average concentration. For the considered time period the average concentration in time integrated sampling $(0.36 \mathrm{mg} / \mathrm{ha})$ was double as when using grab sampling $(0.17 \mathrm{mg} / \mathrm{ha})$.

\subsection{Sampling techniques}

Active sampling utilises instrumentation (often a pump connected to an adsorbent tube or an impinger) and is thus relying on access to an energy source. Fortunately modern instrumentation has low energy consumption and usually batteries are sufficient for 24 hours of sampling. In passive sampling the mass transfer process is depending on diffusion and the pollutants are collected from the sample (air or a liquid) on an adsorbent or in a liquid after transfer across a membrane.

Active sampling with superior control over the flow rate is considered to be more reliable. In passive sampling there is a problem to calibrate the system, since diffusion and at the end the pollutant signal varies with flow-rates in sampling of liquids and wind speed and temperatures in air sampling. However, the inheriting disadvantage of using passive sampling can at least in sampling from liquids be largely eliminated by adding a suitable internal standard early in 
the analytical procedure. In air sampling there is often given vales for the variation of the diffusion coefficient with time so combined with a temperature measurement fairly reliable values can be obtained. Our measurements of solvents in air are that active sampling with active carbon in adsorbent tubes has given 10-20\% higher values than commercially available diffusion samplers. However, passive sampling, especially in connection with sample work-up might be a much simpler and cheaper approach (only one good membrane pump will cost 15-20 000 SEK). For example in the hollow fibre set-up shown below more than 20 samples can easily be handled simultaneously, and the cost for a fibre is below 0.5 SEK.

Figure 2 shows a simple set-up for sampling and (almost) simultaneous work-up of drugs in sewage water.

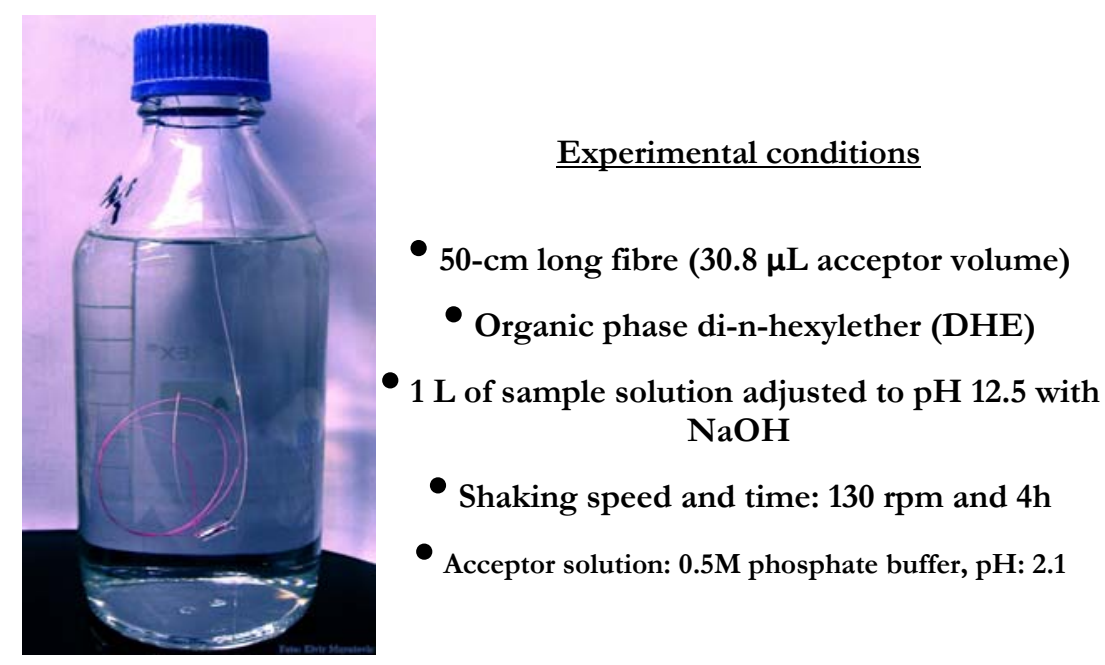

Figure 2. Simple set-up for sample work up of the pharmaceutical fluoxetin [3].

\subsection{General sampling problems}

Two important problems may occur in trace anlysis of pollutants. One of these problems is that the analyte might be unstable. The other is that the concentration of the analyte is so low that contamination or losses further downstream the analytical procedure are difficult to avoid. If, as mentioned above, sampling can be combined with sample-work up these two problems are easier to handle.

For liquid samples membrane technology can be used to create a stable environment for the analyte on the collection side of the membrane and with a stagnant collection solution it is also possible to increase analyte concentration. This is illustrated by figure 2 . An increase in 
analyte concentration can also be achieved using SPE (solid phase extraction) cartridges. However, the feature of increasing the stability of the analyte by exchanging the matrix is more difficult to achieve in SPE, since the disturbing substances might be trapped on the adsorbent column as well. However, in recent studies using so called MEPS (Micro Extractions in Packed Syringes), which is performed using small amounts of solid packing, the analyte may in a short time period be separated from its matrix of plasma or blood (refs 2010). However, here the time between sampling and analysis may be critical. Even with the short analysis times (2-3 minutes) possible using LC-MS-MS with the instrument hooked up to an auto-sampler containing 100 samples it will take 5-6 hours before the last samples are processed.

Instead of transferring unstable anlytes from the sample matrix to another matrix, where the analytes are stable which as mentioned above can be done using membrane technique, perhaps the most common way is to add some chemicals for preservation during the sampling event. For example it is quit common that an acid is added to an aqueous sample aimed for metal analysis. This will work nicely as long as total determination of the metal is the goal. However, if speciation is the objective, one must be aware of that there is for many metals equilibrium between different oxidation stages which is $\mathrm{pH}$-dependent. This means for example that addition of acid will transform $\mathrm{Cr}$ (VI) to $\mathrm{Cr}$ (III) in very short time making analytical determination of $\mathrm{Cr}$ (VI) impossible. The problem of changes in species concentration with time is illustrated in figure 3.
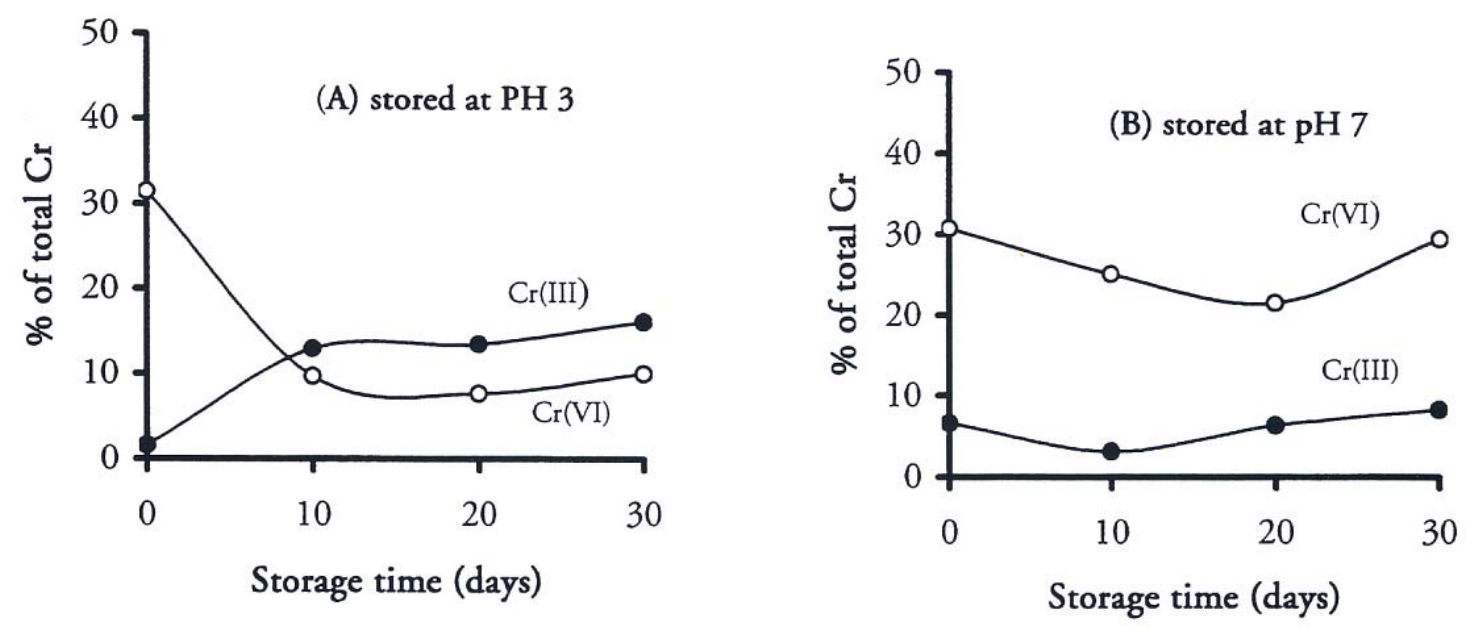

Figure 3. Effect of sample treatyment on $\mathrm{Cr}$ (III) and $\mathrm{Cr}$ (VI) concentration during storage. Sample A was adjusted to $\mathrm{pH} 3$ immediately after collection while B was stored at the natural pH (7.0) [3]. 
A third way to handle the problem with unstable anlytes is to transform them to stable products already in the sampling step. This is illustrated in sampling of analytes containing very reactive isocyanate functions. See figure 4.

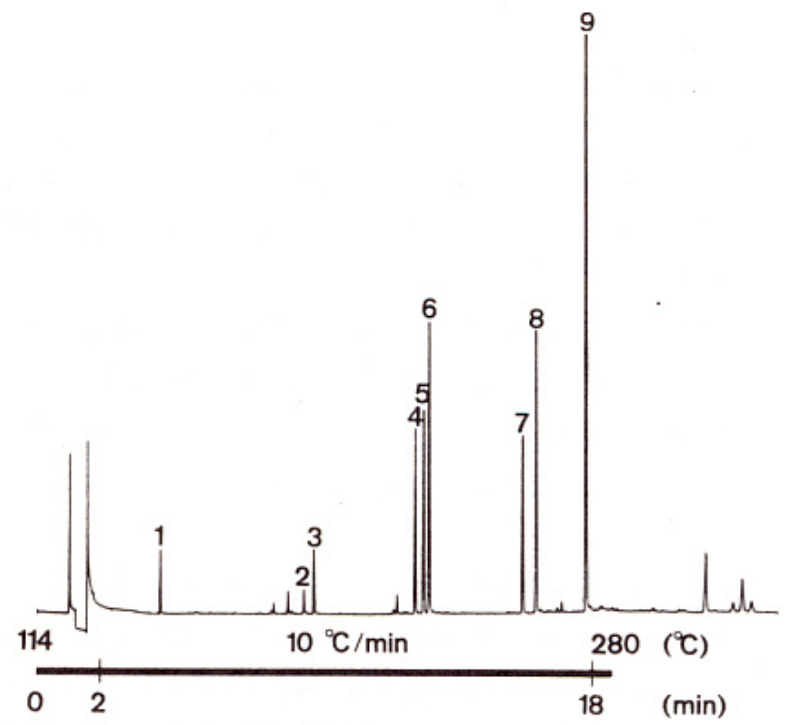

Figure 4. Chromatogram of derivatized thermal degradation products from a polyurethane polymer (Adiprene). Amine groups derivatized with pentaflouroproponic anhydride and isocyanate groups with ethanol. On-column injection of $1 \mu$ toluene solution of derivates of (1) o-chloroanaline, (2) 2,6-TDA, (3) 2,4 TDA, (4-6) aminoisocyanates, (7) 2,6-TDI, (8) 2,4TDI and 3,3'-dichloro-4,4'-diaminodiphenylmethane. [4].

The rapid reaction between isocyanates and ethanol in basic environment using an impinger solution with alkaline ethanol for sampling transfers isocyante functions to corresponding ethyl carbamates. In a subsequent derivatisation step with anhydride of remaining stable amine functions the result is analyte derivatives suitable for GC-MS determination

In air sampling of reactive organic pollutants it is also quite common to use tubes with chemical impregnated adsorbents. This is for example the case when sampling aldehydes, where adsorption tubes impregnated with (diphenyl hydrazine) are commercially available.

\subsection{Increasing the precision and accuracy of the whole analytical procedure}

The most important step to increase precision, and generally also accuracy, when sampling dirty samples is to consider how to compensate for losses that may occur during the whole analytical procedure. A key may then be to add a suitable standard already in connection with the sampling procedure. The best standard would then be a molecule, which in all respects 
behaves like the anlyte considered without being this molecule. With final analysis based on LC-MS or GC-MS a natural choice is a deuterated analog to the analyt or a compound, where some of the carbon-12 atoms in the analyte have been substituted with carbon-13. One large problem is then that such molecules may not be available or can only be made at very high costs. For example ca $50 \mathrm{mg}$ of a deuterated oestrogen will cost in the order of $7000 \mathrm{SE}$. Nevertheless, dramatic improvements in the precision can be achieved. For example when we used three corresponding analogs in the determination of three oestrogen hormones the precision for two of them was improved to ca $6 \%$ compared to ca $30 \%$, when only one of the analogs was used as standard for determination of all the three hormones. This type o deuterated (or even better carbon-13 marked) standard will most probably also compensate for degradation of the analyte, which might be a problem especially for pharmaceuticals. However, in many cases the absolutely best molecule is not available as standard. Then it is important to validate the chosen standard by analysing realistic samples with known added concentrations of anlyte as well as standard, i.e using standard addition technique. Generally if not MS is available, and the samples need to analysed using techniques like LC-UV one should always consider the standard addition technique, i.e. adding known concentrations of the analytes to all samples to be analysed. Of course, one problem then is that each sample needs to be analysed at least three times.

\subsection{Validation of the accuracy}

In many cases the samples after sampling need to be sent away for analysis. To have at least some control over this situation it is well worth to spend extra money to include at least two standard additions with different concentrations of the analytes to be determined. That will give you an indication of how well the methodology used in the analytical laboratory works for your types of samples. A close study of published results concerning pollutants in waste waters reveals that this measure often not seems to be taken. Another way of dealing with the validity problems may of course be to send the samples to two laboratories but in this case an extra uncertainty may arise due to differences in the transport routes, and different time gaps between sampling and sample analysis.

\section{CONCLUDING REMARKS}

From the evidence given in this paper it is obvious that when considering the given problem which demands analytical measurements, one should always start by carefully evaluating the sampling situation. This might in many cases decrease the risk that analytical results obtained will be of no use for the decision maker, and will also in many cases most likely simplify the total analytical procedure, thus saving time and money.

\section{REFERENCES}


[1] Bergström, S., Svensson, B-M., Mårtensson, L., and Mathiasson, L. 2007 Development and application of an analytical protocol for evaluation of treatment processes for landfill leachates. I. Development of an analytical protocol for handling organic compounds in leachate samples. Int. J. Environ. An. Ch. 87, 1-15.

[2] Mathasson, L., Nilvé, G. and Ulén, B 1991. A liquid membrane inrichment technique for integrating field sampling in water applied to MCPA. Int. J. Environ. An. Ch. 45, 117-125.

[3] Ndungù, K. 1999. Liquid membrane extraction techniques for trace metal analysis and speciation in environmental and biological matrices. Doctoral dissertation, Department of Analytical Chemistry, Lund University, Sweden.

[4] Zorita, S., Mårtensson, L. and Mathiasson, L. 2007 A hollow-fibre liquid membrane extraction for determination of fluoxetine and norfluoxetine concentrations at ultra trace level in sewage samples. J. Sep. Sci. 30, 2513-2521.

[5] Skarping, G., Renman, L., Sangö, C., Mathiasson,L. and Dalene M. 1985. Capillary chromatographic method for the determination of complex mixture of isocyanates and amines. J. Chromatogr 346, 191-204. 